\title{
Evaluación del consumo de riesgo de alcohol en estudiantes universitarios de la Facultad de Farmacia
}

\section{Alcohol consumption in college students from the pharmacy faculty}

\author{
Laia Miquel*******, Miquel Rodamilans****, Rosa Giménez****, Trinitat Cambras****, \\ Anna Maria Canudas****, Antoni Gual*,**,***
}

*Grup de recerca en Addiccions Clínic, Unitat de conductes addictives. Hospital Clínic Universitari de Barcelona, Institut Clínic de Neurociencies, España. **IDIBAPS. ***Red de Trastornos Adictivos (RTA), RETICS. ****Grupo de Trabajo Colaborativo Casos Clínicos Transversales de la Facultad de Farmacia (CCT-FARMA), Universidad de Barcelona, España.

\section{Resumen}

El consumo de alcohol es muy prevalente entre los estudiantes universitarios. La detección precoz en futuros profesionales sanitarios es muy importante puesto que puede incidir no solo en su salud, sino también en su enfoque en futuras acciones preventivas como profesionales de la salud.

Detectar la prevalencia del consumo de riesgo de alcohol en estudiantes de farmacia y en el profesorado y comparar el patrón de consumo entre estos grupos, y según el curso académico.

Se realiza un cribado del consumo de alcohol mediante el cuestionario AUDIT (Alcohol Use Disorders Identification Test) a estudiantes universitarios de farmacia $(n=434)$ en el marco de un proyecto de innovación docente. Se realiza un análisis comparativo entre los estudiantes de primero y quinto curso, y el profesorado.

El 31,3\% de los estudiantes fueron identificados como bebedores de riesgo. La mayor prevalencia de consumidores de riesgo y las mayores puntuaciones totales se observaron en los alumnos de primer curso. Los estudiantes de primero de turno de mañana presentaron un riesgo de 1,9 (IC 95\% 1,1-3,1) comparado con los de quinto. La frecuencia de consumo de alcohol se incrementa con el curso académico, mientras que el número de consumiciones por día de consumo se reduce. Durante el primer año en la Facultad los estudiantes presentan una elevada prevalencia de consumo de riesgo. Puesto que con la edad se observa una tendencia decreciente en dichos consumos, es importante diseñar intervenciones preventivas que la favorezcan. Palabras clave: AUDIT cuestionario, cribado, alcohol, consumo de riesgo, estudiantes universitarios.

\section{Abstract}

Alcohol consumption is highly prevalent in university students. Early detection in future health professionals is important: their consumption might not only influence their own health but may determine how they deal with the implementation of preventive strategies in the future.

The aim of this paper is to detect the prevalence of risky alcohol consumption in first- and last-degree year students and to compare their drinking patterns.

Risky drinking in pharmacy students $(n=434)$ was assessed and measured with the AUDIT questionnaire (Alcohol Use Disorders Identification Test). A comparative analysis between college students from the first and fifth years of the degree in pharmacy, and that of a group of professors was carried to see differences in their alcohol intake patterns.

Risky drinking was detected in $31.3 \%$ of students. The highest prevalence of risky drinkers, and the total score of the AUDIT test was found in students in their first academic year. Students in the first academic level taking morning classes had a two-fold risk of risky drinking (OR=1.9 (IC 95\%1.1-3.1)) compared with students in the fifth level. The frequency of alcohol consumption increases with the academic level, whereas the number of alcohol beverages per drinking occasion falls.

Risky drinking is high during the first year of university. As alcohol consumption might decrease with age, it is important to design preventive strategies that will strengthen this tendency.

Keywords: AUDIT questionnaire, screening, alcohol, hazardous drinking, college students. 
$\mathrm{E}$ n los últimos años, el consumo de alcohol entre la población joven está experimentando un crecimiento. En especial, el patrón en forma de atracón y las consultas en urgencias por intoxicación etílica, frecuentes sobretodo en las mujeres ("Observatorio Español de la droga y las toxicomanías," 2011). Los estudiantes universitarios son, especialmente, una población de riesgo (Karam, Kypri y Salamoun, 2007) y esta etapa es un buen momento para introducir estrategias preventivas.

Aproximadamente entre un 20-60\% de la población universitaria presenta consumo de riesgo (Arrieta, 2009; Montaño, Morales, Gómez, Maldonado y Gantiva, 2011; Pengpid, Peltzer, van der Heever y Skaal, 2013). El elevado consumo de bebidas alcohólicas en jóvenes se ha asociado con un elevado índice de mortalidad, accidentalidad y conductas de riesgo como conducir bajo los efectos del alcohol, mantener relaciones sexuales sin protección o problemas familiares (Barlés, Escario, Galbé, 2014; Arrieta, 2009; Hingson, Heeren, Winter y Wechsler, 2005). Algunos de los factores de riesgo relacionados con el consumo excesivo de alcohol son: el ser varón, la impulsividad, el fácil acceso al dinero, vivir solo o iniciar el consumo a una edad temprana (Cortés, Giménez, Motos y Cadaveirea, 2014; Montaño et al., 2011; Mota et al., 2010; Restrepo, Agudelo, Giraldo y Sánchez, 2011; Wicki, Kuntsche y Gmel, 2010). Muchos estudios han abordado este problema en estudiantes universitarios. La mayoría de estos se centran en aspectos sociológicos (Bani et al., 2013; Wicki et al., 2010; Young y de Klerk, 2008) y factores de riesgo asociados (Vinader-Caerols, Monleaon y Parra, 2014; Ansari, Stock y Mills, 2013; Caamaño-Isorna, Corral, Parada y Cadaveira, 2008; DeMartini y Carey, 2012; Karam et al., 2007). Sin embargo, existe poca información sobre los cambios en el patrón de consumo en función del curso académico y de las diferencias entre estudiantes y profesorado. No se han llevado a cabo trabajos de seguimiento a lo largo de los estudios universitarios, ni evaluación de programas de intervención precoz durante los mismos. Además, los datos en relación a la prevalencia del consumo de riesgo son bastante heterogéneos debido a los diferentes instrumentos de medida utilizados y las definiciones empleadas por los distintos autores.

La sensibilización y prevención del consumo de alcohol en población adulta joven es especialmente relevante. En la actualidad, se dispone de instrumentos de cribado del consumo de riesgo del alcohol de fácil aplicación y, también, de intervenciones terapéuticas (intervención breve presencial (Pengpid et al., 2013) o online (Khadjesari, Murray, Hewitt, Hartley y Godfrey, 2011)) que han demostrado ser eficaces en la reducción del consumo de riesgo (Seigers y Carey, 2011; Toumbourou et al., 2007). Las intervenciones dirigidas a futuros agentes de la salud son especialmente importantes dado que no solamente tienen repercusión en éstos sino también sobre la población en general. La etapa universitaria, que es considerada de especial riesgo, es un buen momento para intervenir de forma precoz. En este sentido, el Grupo de Trabajo Colaborativo Casos Clínicos Transversales de la Facultad de Farmacia de la Universidad de Barcelona (CCT-FARMA), decidió desarrollar, un caso clínico transversal sobre consumo de riesgo de alcohol a lo largo de los cinco cursos del Grado de Farmacia. Se pretendía que valoraran el propio riesgo del consumo excesivo de alcohol y proporcionarles herramientas para reconocer e identificar conductas de riesgo en grupos de proximidad que les permitan realizar intervenciones breves en su futura praxis profesional(Giménez et al., 2013; Rodamilans et al., 2012).

El objetivo de este estudio es tener una visión global del patrón de consumo de alcohol de los estudiantes de la Facultad de Farmacia de la Universidad de Barcelona mediante la determinación de la prevalencia del consumo de riesgo en estudiantes de primero y quinto curso previo al desarrollo del proyecto CCT-FARMA. Al mismo tiempo se valora el riesgo de los profesores participantes del Grupo de Trabajo, con la finalidad de comprobar el grado de sensibilización de los mismos al abordar un caso clínico sobre consumo de riesgo.

También se pretende comparar las diferencias en el patrón de consumo entre los alumnos de estos cursos académicos y el profesorado tras obtener los datos de consumo de alcohol de manera transversal en el momento que se inicia el proyecto docente.

\section{Método}

\section{Diseño}

En el año 2011-2012, de forma transversal, se valoró el patrón de consumo de alcohol de los estudiantes de primer y quinto curso, y también del profesorado.

\section{Población}

Se seleccionaron aquellos individuos que estaban realizando estudios de farmacia en la Universidad de Barcelona (UB). Se recogieron datos de los estudiantes de primero (turno de mañana y turno de tarde), quinto y del profesorado. De los 434 alumnos de primer curso matriculados respondieron a las encuestas anónimas un 67,1\%; de los 325 alumnos de quinto participó un $34,2 \%$ y de un total de 46 profesores implicados en el proyecto educativo respondió el $84,8 \%$.

\section{Procedimiento}

En el año 2012, se inició el proyecto de innovación docente (CCT-FARMA), en el que se introdujo este caso transversal, empezando por los alumnos de primer curso. Este caso clínico se aborda desde los distintos puntos de vista que proporcionan las asignaturas del grado de Farmacia (Rodamilans et al., 2012). Para evaluar la eficacia de CCT-FARMA se establecieron mecanismos de cuantificación académicos (conocimientos, habilidades, capacidad de integración y de 
interpretación) y de tipo sanitario como la valoración del consumo de riesgo de alcohol (test AUDIT). De forma sorpresiva, durante el horario lectivo, se solicitó a los estudiantes que respondieran de forma voluntaria y anónima al test para la identificación de los trastornos debidos al consumo de alcohol (AUDIT). El profesorado respondió el test el mismo día, de la misma forma.

Los resultados preliminares presentados forman parte del nuevo proyecto formativo (CCT-FARMA), uno de cuyos objetivos es evaluar al final del Grado de Farmacia y mediante el test AUDIT, si el desarrollo de este caso clínico modifica el consumo de alcohol en referencia a esta evaluación inicial del año 2012.

\section{Instrumentos de recogida de datos}

Como instrumento de cribado del consumo de riesgo de alcohol en la población universitaria se utilizó la versión completa del test para la identificación de los trastornos debidos al consumo de alcohol (AUDIT). Este instrumento estandarizado, desarrollado por la Organización mundial de la Salud, consta de 10 preguntas que interrogan sobre la cantidad, la frecuencia y las consecuencias del consumo de alcohol. Está validada en español y catalán, y su nivel de fiabilidad es buena (Alpha de Cronbach=0,89) (Contel Guillamón, Gual Solé y Colom Farran, 1999) también para poblaciones universitarias (Fleming, Barry y MacDonald, 1991). El test de cribado ha demostrado tener buena sensibilidad y especificidad, no solamente para detectar el consumo perjudicial y de riesgo sino también para los trastornos por uso de alcohol. Utilizando como punto de corte 8 , el test tiene una sensibilidad y especificidad del $90 \%$ y $61 \%$ (Barry y Fleming, 1993). Según el estudio de Contel y cols (1991) en el grupo de hombres, si utilizamos como punto de corte el 9, la sensibilidad del AUDIT es del 90\% y la especificidad del $81,5 \%$. Y en el grupo de mujeres (punto de corte 6 ) la sensibilidad es del $33,3 \%$ y la especificidad del $91,6 \%$.

\section{Análisis estadístico}

Se realizó un análisis descriptivo de los datos sociodemográficos (sexo y edad) del total de la muestra y según el grupo estudiado (primer curso turno de mañana, primer curso turno de tarde, quinto y profesores). Las variables que corresponden a cada pregunta del AUDIT se analizaron como variable cuantitativa y también categórica. Sé verificó el tipo de distribución de la variable AUDIT cuantitativa mediante el test de normalidad Kolmogorov-Smirnov. $\mathrm{Al}$ no seguir una distribución normal se utilizaron pruebas no-paramétricas (Kruskal-Walis) para comparar la puntuación total del AUDIT según el grupo evaluado, además se utilizó ANCOVA de rangos para ajustar por sexo y edad. Para realizar el análisis comparativo 2 a 2 entre cada grupo se utilizó la prueba no-paramétrica U-de Mann Whitman. Para detectar los grupos de consumo de riesgo se decidió categorizar la puntuación total del AUDIT en una variable binaria utilizando como punto de corte igual o superior a 9 en varones y 6 en mujeres (Pérula de Torres et al., 2005; Rubio Valladolid, Bermejo Vicedo, Caballero Sánchez-Serrano y Santo-Domingo Carrasco, 1998). Para analizar variables categóricas se utilizó el test chi-cuadrado y ANOVA para las variables cuantitativas. Las comparaciones múltiples posthoc se realizaron mediante Bonferroni. Se considera estadísticamente significativo cuando $\mathrm{p} \leq 0.05$. Se realizó análisis de correlación de Pearson para determinar la correlación entre la edad y el nivel académico $(r=0.73$, p<0.001). Para analizar la relación entre la presencia de consumo de riesgo y el curso universitario se realizó un análisis de regresión logística utilizando como co-variable el sexo y la edad.

\section{Resultados}

Participaron en el estudio un total de 440 personas. Se obtuvo datos completos de edad y sexo de 434 individuos. Del total de respondedores, 286 estaban matriculados en el primer curso académico de la universidad de Farmacia (204

Tabla 1

Descripción del consumo de riesgo en función del curso académico

\begin{tabular}{|c|c|c|c|c|c|c|c|}
\hline & $\begin{array}{l}\text { PTM } \\
\text { (204) }\end{array}$ & $\begin{array}{l}\text { PTT } \\
(82)\end{array}$ & $\begin{array}{l}\text { Quinto } \\
\text { (111) }\end{array}$ & $\begin{array}{c}\text { Profesores } \\
\text { (37) }\end{array}$ & $\begin{array}{l}\text { Total } \\
\text { (434) }\end{array}$ & & \\
\hline & $\mathrm{n}(\%)$ & $\mathrm{n}(\%)$ & $\mathrm{n}(\%)$ & $\mathrm{n}(\%)$ & $\mathbf{N}(\%)$ & $\chi^{2} / F$ & $p$-valor \\
\hline Sexo (Mujer) & $148(72,5)$ & $62(75,6)$ & $93(83,8)$ & $26(70,3)$ & $329(75,8)$ & 5,7 & 0,13 \\
\hline $\begin{array}{l}\text { Edad } \\
\text { (media (DE)) }\end{array}$ & $18,5(1,1)^{a, b}$ & $19,8(4,0)^{c}$ & $24,2(3,9)^{d}$ & $50,9(7,3)$ & $22,96(9,5)$ & 935,2 & $<0,001$ \\
\hline $\begin{array}{l}\text { AUDIT Total } \\
\text { (media (DE)) }\end{array}$ & $6,3(5,3)$ & $5,3(3,9)$ & $4,6(4,1)$ & $2,6(1,3)$ & $5,35(4,7)$ & & $<0,001$ \\
\hline C. Riesgo & $82(40,2)$ & $25(30,5)$ & $29(26,1)$ & 0 & $136(31,3)$ & 26,3 & $<0,001$ \\
\hline $\begin{array}{l}\text { Hombres }(\%) \\
\text { Mujeres }(\%)\end{array}$ & $\begin{array}{l}26(46,4) \\
56(37,8)\end{array}$ & $\begin{array}{c}5(25,0) \\
20(32,3)\end{array}$ & $\begin{array}{c}6(33,3) \\
23(24,7)\end{array}$ & & $\begin{array}{l}37(35,2) \\
99(30,1)\end{array}$ & 1,0 & 0,32 \\
\hline
\end{tabular}

Nota. PTM: Primero turno de mañana; PTT: Primero turno de tarde; aPTM vs PTT $p=0,039$; ${ }^{\text {PTTM vs }}$ Quinto y vs Profesores $p<0,001 ;$ cPTT vs Quinto vs Profesores $p<0,001 ;{ }^{d}$ Quinto vs Profesores $p<0,001$ 
de turno de mañana y 82 de turno de tarde), 111 en quinto y 37 eran profesores. El 75,8\% de la muestra total que participó en el estudio fueron mujeres, lo cual corresponde a la distribución normal en los estudiantes de esta facultad. No se observaron diferencias significativas en la distribución del sexo según el curso académico ni tampoco en el profesorado (Tabla 1). La edad media de toda la muestra fue de 23,0 (DE $9,5)$ años, siendo las diferencias en las edades medias de cada grupo estudiado estadísticamente significativas (Tabla 1).

\section{Relación entre el patrón de consumo de alcohol y el curso académico}

El 31,3\% de los estudiantes presentó criterios de consumo de riesgo. La mayor proporción de bebedores de riesgo se observó en los estudiantes del turno de mañana de primer curso. El 40,2\% de los estudiantes de primero de turno de mañana presentaron consumo de riesgo, seguido de los del turno de tarde $(30,5 \%)$ y los de quinto $(26,1 \%)$. Entre el profesorado no se detectaron consumidores de riesgo (Tabla 1). Tras ajustar los resultados por la variable sexo, se observó que los estudiantes de primero de turno de mañana tenían una razón de probabilidad casi el doble de presentar consumo de riesgo comparado con los estudiantes de quinto $(\mathrm{OR}=1,9$ IC95\% 1,1-3,1) (Tabla 2). Si ajustamos los datos por edad, las diferencias entre los cursos pierden significación estadística porque a más edad disminuye el riesgo de consumo de riesgo ( $\mathrm{OR}=0,84 \mathrm{IC} 95 \%$ 0,72-0,98) (Tabla 2).

Las figuras 1 y 2 muestran la frecuencia y cantidad de consumo de alcohol de los diferentes grupos estudiados. Los datos mostraron la presencia de diferencias significativas entre

Tabla 2

A. Relación entre el curso académico y el riesgo de consumo de alcohol tras ajustar por sexo

\begin{tabular}{cccc}
\hline & & \multicolumn{2}{c}{ 95\% I.C. } \\
& OR & Min & Max \\
\hline PTM vs PTT & 1,5 & 0,9 & 2,6 \\
PTM vs quinto & 1,9 & 1,1 & 3,1 \\
PTT vs quinto & 1,2 & 0,6 & 2,3 \\
Sexo & 1,3 & 0,8 & 2,0 \\
\hline
\end{tabular}

Nota. PTM: Primero turno de mañana; PTT: Primero turno de tarde

B. Relación entre el curso académico y el riesgo de consumo de alcohol tras ajustar por edad y sexo

\begin{tabular}{cccc}
\hline & & \multicolumn{2}{c}{ 95\% I.C. } \\
& OR & Min & Max \\
\hline PTM vs PTT & 1,3 & 0,8 & 2,3 \\
PTM vs quinto & 0,7 & 0,3 & 1,7 \\
PTT vs quinto & 0,5 & 0,2 & 1,4 \\
Sexo & 1,4 & 0,9 & 2,3 \\
Edad & 0,8 & 0,7 & 0,98 \\
\hline
\end{tabular}

Nota. PTM: Primero turno de mañana; PTT: Primero turno de tarde los grupos tanto en relación a la frecuencia de consumo $\left(\chi^{2}=\right.$ 33,2; $\mathrm{p}<0,001)$ como en la cantidad de bebidas alcohólicas ingeridas en un día normal $\left(\chi^{2}=68,4 ; p<0,001\right)$. En general, la frecuencia de consumo más habitual que realizó el total de la muestra fue la toma de alcohol de 2 a 4 veces al mes $(54.8 \%)$ seguido del consumo mensual $(22,4 \%)$. Tan solo un 6,2\% nunca bebió en el último año. Los profesores fueron el grupo que bebió con más frecuencia (43,2\%: más de

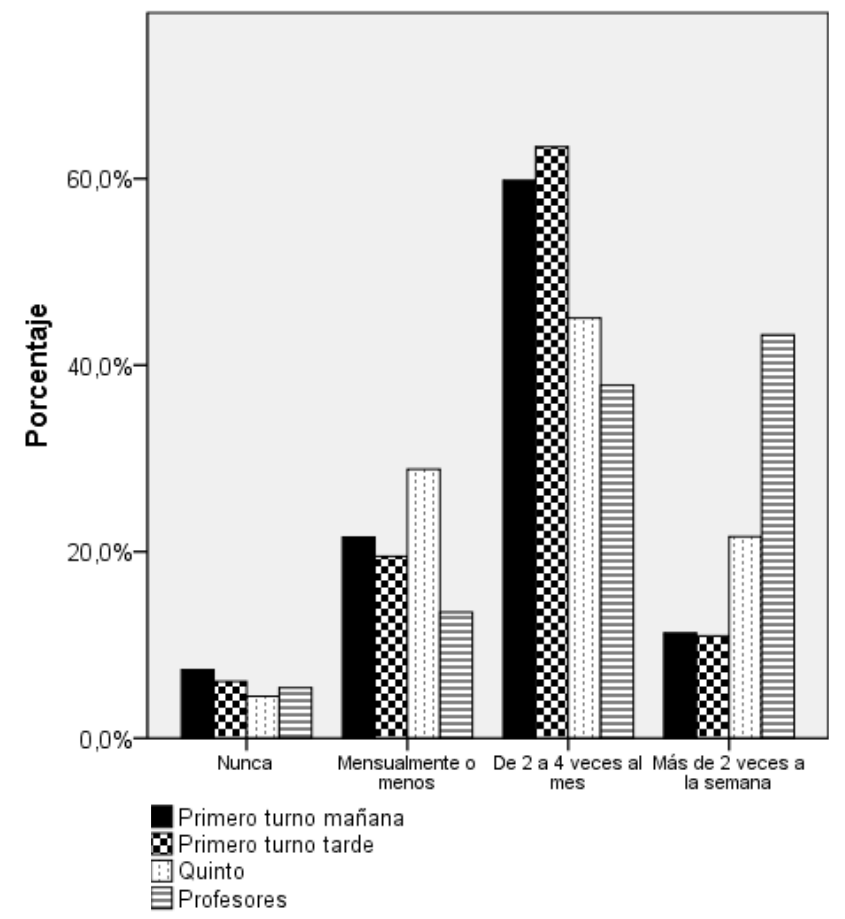

Figura 1. ¿Con qué frecuencia consume alguna bebida alcohólica?

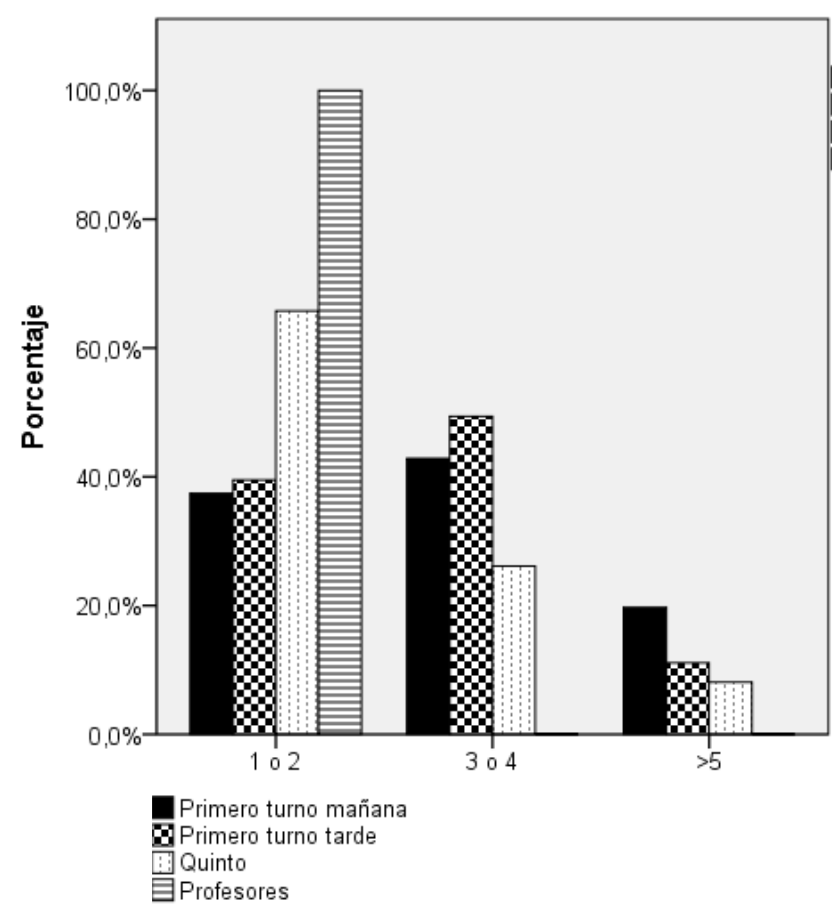

Figura 2. ¿Cuántas consumiciones de bebidas alcohólicas suele realizar en un día de consumo normal? 


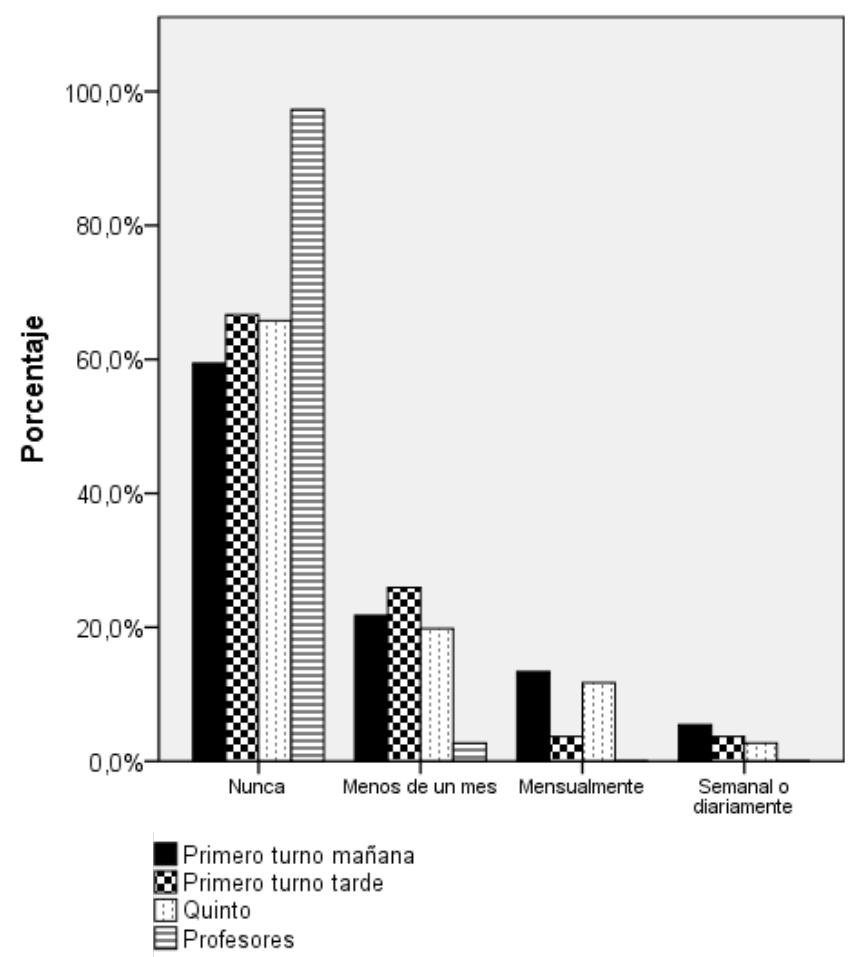

Figura 3. ¿Con qué frecuencia toma 6 o más bebidas alcohólicas en un solo día?

2 veces a la semana) seguido de los de quinto curso $(21,6 \%)$ y los de primero de turno de mañana $(11.3 \%$ ) (Figura 1). La mayoría de alumnos de primero $(59,8 \%$ turno de mañana, $63,4 \%$ turno de tarde) y quinto $(45,0 \%)$ bebieron de 2 a 4 veces al mes (Figura 1).

En el caso del profesorado, la cantidad bebida por ocasión de consumo sigue una tendencia diametralmente opuesta a la frecuencia de los consumos. Así, aunque consumen alcohol con una mayor frecuencia (42,3\% más de 2 veces a la semana) (Figura 1) la cantidad de alcohol ingerida por ocasión es baja ( 1 o 2 consumiciones por ocasión de consumo) (Figura 2). En el caso de los estudiantes se observa como la cantidad de alcohol consumida decrece a medida que pasan los cursos. Así, la mayor proporción de estudiantes que beben más de 5 consumiciones por ocasión de consumo se concentra en el grupo de primero de turno de mañana $(19,7 \%)$ seguido de los del turno de tarde $(11,1 \%)$ y los de quinto $(8,1 \%$ ) (Figura 2). En este sentido, también se observa que los alumnos de primer curso de turno de mañana fueron los que tomaron con mayor frecuencia 6 o más bebidas alcohólicas (Figura 3). La mayoría de alumnos de éste curso cuando ingieren más de 6 bebidas por ocasión lo hacen mensualmente o menos $(35,2 \%)$ y un $5,4 \%$ realizan consumos elevados semanalmente o más.

\section{Comparación entre la puntuación total del AUDIT y el curso académico}

Se observaron diferencias estadísticamente significativas al comparar la puntuación total de los 4 grupos estudiados

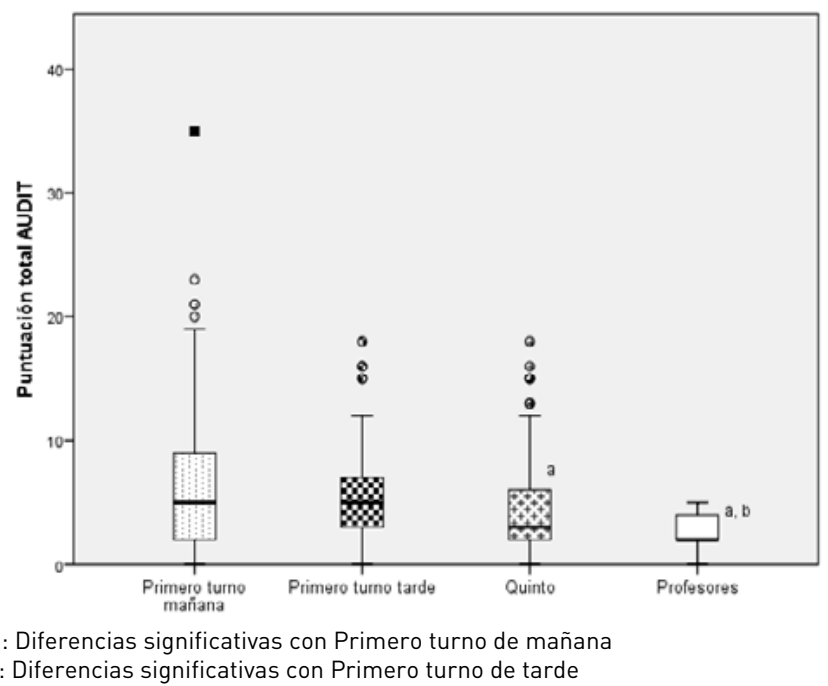

Figura 4. Puntuación Total del cuestionario AUDIT según el curso académico

$(\mathrm{p}<0,001)$ (Figura 4, Tabla 1). Tras ajustar por edad y sexo la relación entre la puntuación total del AUDIT y el nivel académico perdió significación estadística $(\mathrm{F}=1,138$; $\mathrm{p}=$ 0,334). Los profesores presentaron una puntuación total media significativamente inferior a los individuos de primer curso (diferencia media con turno de mañana: 3,7 puntos; $\mathrm{p}<0,001$; diferencia media con turno de tarde: 2,7 puntos $\mathrm{p}<0,001)$. También se observaron diferencias estadísticamente significativas entre los respondedores de primero de turno de mañana y los de quinto curso $(\mathrm{p}=0,003)$, siendo la media obtenida superior en el curso de primero (diferencia media $=1,7$ puntos).

\section{Discusión}

Los primeros resultados del estudio de evaluación de la eficacia del proyecto CCT-FARMA que muestran datos de cribado del consumo de riesgo en la población universitaria de la facultad de Farmacia de Barcelona (31,3\%), nos indican que un porcentaje importante de los estudiantes se podría beneficiar de una intervención con el objetivo de reducir el consumo entre los alumnos y así concienciar a los futuros agentes sanitarios de los riesgos del consumo de alcohol. En resumen, el consumo de riesgo y la puntuación total del AUDIT se reduce a medida que aumenta el curso académico de manera que los estudiantes de primero de turno de mañana presentan un mayor consumo de riesgo y puntuación media del AUDIT total comparado con el curso superior. El patrón de consumo entre los estudiantes y el profesorado es contrapuesto. Los alumnos tienen una mayor tendencia a realizar ingestas de alcohol elevadas por ocasión de forma episódica mientras que los profesores beben menor cantidad con mayor frecuencia.

La prevalencia de consumo de riesgo en la muestra universitaria $(31,3 \%)$ es bastante más elevada que la descrita en 
población general $(10 \%)$, pero similar a otras poblaciones universitarias de otros países (Ansari et al., 2013; Caamaño-Isorna et al., 2008; Pengpid et al., 2013; Reavley, Jorm, McCann y Lubman, 2011; Young y de Klerk, 2008)

Aunque el porcentaje de varones con consumo de riesgo fue ligeramente superior al de mujeres, tales diferencias no fueron significativas a diferencia de lo que se ha observado en otros estudios (Ansari et al., 2013; DeMartini y Carey, 2012; Reavley et al., 2011). En este sentido cabe destacar que a diferencia de otros investigadores tuvimos en cuenta diferentes puntos de corte para hombres y mujeres. Aunque según Wicki M y cols. (2010) estas diferencias de género se mantienen incluso cuando se utilizan puntos de corte distintos de consumo de riesgo para hombres y mujeres.

El grupo de alumnos universitarios que tiene más bebedores de riesgo es el de primero de turno de mañana (42\%) seguido de los de turno de tarde $(30,5 \%)$ y los de quinto $(26,1 \%)$. Aunque nuestros resultados coinciden con otros autores (Ansari et al., 2013; Sebena, Orosova, Mikolajczyk y van Dijk, 2011) existe cierta controversia en relación a la asociación que existe entre la edad y el riesgo de consumo de alcohol. Mientras algunos estudios han observado un incremento del riesgo a mayor edad o curso académico, otros estudios no observan ninguna relación o lo que es más frecuente que ésta sea negativa (Sebena et al., 2011; Wicki et al., 2010). No se detectan diferencias significativas entre los alumnos de primero de turno de mañana y de turno de tarde posiblemente porque los dos grupos tienen edades similares, aunque se desconoce si existen otros factores diferenciales que puedan intervenir, como por ejemplo diferencias en los motivos por los cuales unos alumnos están matriculados en un horario u otro. Nuestros datos sugieren que las diferencias entre los cursos académicos son debidas a las diferencias de edad. Contrariamente a lo que ocurre en otros países, el consumo de riesgo en el profesorado fue nulo (Reavley et al., 2011). Los resultados obtenidos mediante el test AUDIT al principio de este proyecto (2012), nos muestran que se produce una reducción del riesgo entre los alumnos de primero y quinto curso. El paso por la Facultad por tanto puede constituir una buena oportunidad para potenciar esta tendencia a la reducción de consumos de riesgo, mediante intervenciones de concienciación y sensibilización.

El patrón de consumo de los estudiantes de farmacia es opuesto al de los profesores de la misma facultad. Los alumnos tienen un consumo más esporádico (mayoritariamente consumen de 2 a 4 veces al mes) y consumen mayores cantidades de alcohol por ocasión (más de 3 bebidas), mientras que el profesorado consume entre 1 o 2 bebidas alcohólicas con una frecuencia superior a dos veces a la semana $(43,2 \%)$. Este mismo patrón coincide con el de otros estudios (Reavley et al., 2011; Slutske, 2005).

Uno de los principales problemas de los estudiantes universitarios es el consumo en forma de Binge drinking (consumo compulsivo o en atracón) que ha sido ampliamente descrito por varios autores (Ansari et al., 2013; Jim McCambridge et al., 2013). Entre un 34,2-40,6\% de los alumnos de farmacia realizaron en algún momento del año previo a la encuesta un consumo de más de 6 bebidas alcohólicas en un día, coincidiendo con los datos poblacionales españoles (EDADES 2011). En España, se observa un descenso de este tipo de consumo con la edad, siendo la prevalencia de esta forma de consumo más elevada entre las edades de 20-24 años (EDADES 2011), coincidiendo con la etapa de formación universitaria. En algunos estudios europeos el porcentaje de consumidores excesivos (de 5 o más bebidas) se sitúa alrededor del 60-70\% (Ansari et al., 2013; Dantzer, Wardle, Fuller, Pampalone y Steptoe, 2006). Los estudiantes beben en forma de atracón con mayor frecuencia que el profesorado igual que ocurre en otros países (Reavley et al., 2011). Sin embargo, comparado con el estudio realizado por Reavley y cols. (2011) donde se objetivó que el $21 \%$ del equipo docente bebía más de 6 consumiciones al mes o más, los de nuestra muestra refirieron no ingerir más de 6 bebidas con una frecuencia superior a la mensual.

Entre las principales limitaciones del estudio debemos destacar que los datos relacionados con el consumo de alcohol se obtienen a partir de datos declarados, que aunque son fiables en poblaciones clínicas, en población general podrían estar sesgadas, a pesar de haber respondido al test de forma anónima. Tampoco se recogieron algunos datos sociodemográficos que podrían actuar de factor de riesgo, ni tampoco aspectos de tipo sociocultural que podrían sesgar los resultados. Además se debe tener en cuenta los resultados de este estudio se han obtenido en una muestra de universitarios y que por tanto los datos podrían no ser extrapolables a otras poblaciones no universitarias.

La elaboración del caso clínico sobre consumo de riesgo de alcohol tratado desde diferentes asignaturas del grado de farmacia, supone una estrategia de enseñanza con finalidad integradora. Para ello, se diseñó el caso clínico sobre consumo de riesgo de alcohol, a través de un personaje ficticio, Sam, que clínicamente fue validado por la Unidad de Conductas Adictivas ajustándose a casos reales. Posteriormente se coordinaron las asignaturas participantes. La patología de este personaje evoluciona a lo largo de los cinco cursos del Grado de Farmacia, y permite a los estudiantes, en cierto modo, seguir de cerca la vida del personaje. Para aumentar la concienciación de los alumnos y considerar que el consumo de riesgo de alcohol no está muy alejado de su entorno, se utiliza la evaluación del consumo de riesgo del propio estudiante.

Campañas preventivas en esta población diana pueden resultar muy beneficiosas dado el importante factor social y de normas por el cual se rige esta población (Wicki et al., 2010). Intervenir en la población universitaria de riesgo puede aportar importantes beneficios, no solamente en los resultados académicos de éstos dado que justamente son los alumnos menos motivados para tener un buen rendimiento académico (Ansari et al., 2013) sino para evitar conductas 
de riesgo de tipo sexual y la accidentalidad. Puesto que las intervenciones breves (incluidas las virtuales) en esta población son efectivas (Bewick et al., 2013; Jim McCambridge et al., 2013; Pengpid et al., 2013; Seigers y Carey, 2011), es importante poder detectar el consumo de riesgo con precocidad con el fin de modificar los patrones de consumo en una población tan vulnerable a sus efectos. Sin ninguna duda, los nuevos desarrollos preventivos deben hacer uso extensivo de las nuevas formas de comunicación(J McCambridge, Bendtsen, Bendtsen y Nilsen, 2012).

\section{Reconocimientos}

Proyecto de innovación docente (2011PID-UB/19 y 2012PID/UB/157) Universitat de Barcelona.

Proyecto RD12/0028/0016, integrado en el Plan Nacioal de I+D+I y cofinanciado por el ISCII-Subdirección General de Evaluación y el Fondo Europeo de Desarrollo Regional (FEDER).

\section{Conflicto de intereses}

El Dr. Antoni Gual ha recibido subvención económica de Lundbeck, DyA Pharma y TEVA así como honorarios de parte de Lundbeck, DyA Pharma y Abbivie durante el estudio que no tienen relación con el trabajo presentado.

La Dra. Laia Miquel ha recibido honorarios de Lundbeck que no tienen relación con éste trabajo.

El resto de autores no presentan conflicto de intereses para el estudio.

\section{Referencias}

Ansari, W. El, Stock, C., y Mills, C. (2013). Is Alcohol Consumption Associated with Poor Academic Achievement in University Students? International Journal of Preventive Medicine, 4, 1175-1188.

Arrieta, K. (2009). Consumo patológico de alcohol entre los estudiantes de la Universidad de Cartagena. Revista de Salud Pública, 11, 878-886.

Bani, R., Hameed, R., Szymanowski, S., Greenwood, P., Kribs-Zaleta, C., y Mubayi, A. (2013). Influence of environmental factors on college alcohol drinking patterns. Mathematical Bioscience and Engineering, 10, 1281-1300.

Barlés Arizon, M. J., Escario, J. J. y Galbé Sáncehz-Ventura, J. (2014). Predictors of driving under the inluence of alcohol among Spanish adolescents. Adicciones, 26, 96-405.

Barry, K. L. y Fleming, M.F. (1993). The Alcohol Use disorders Identification Test (AUDIT) and the SMAST-13: predictive validity in a rural primary care sample. Alcohol and alcoholism, 28, 33-42.

Bewick, B. M., West, R. M., Barkham, M., Mulhern, B., Marlow, R., Traviss, G., y Hill, A. J. (2013). The effectiveness of a Web-based personalized feedback and social norms alcohol intervention on United Kingdom university students: randomized controlled trial. Journal of Medical Internet Research, 15, e137. doi:10.2196/jmir.2581.

Caamaño-Isorna, F., Corral, M., Parada, M., y Cadaveira, F. (2008). Factors associated with risky consumption and heavy episodic drinking among Spanish university students. Journal of Studies on Alcohol and Drugs, 69, 308-312.

Contel Guillamón, M., Gual Solé, A., y Colom Farran, J. (1999). Test para la identificación de transtornos por uso de alcohol (AUDIT): Traducción y validación del AUDIT al catalán y castellano. Adicciones, 11, 337-347.

Cortés, M., Giménez, J. A., Motos, P. y Cadaveira, S . (2014). The importance of expectations in the relationship between impulsivity and binge drinking among university students. Adicciones, 26, 134-145.

Dantzer, C., Wardle, J., Fuller, R., Pampalone, S. Z., y Steptoe, A. (2006). International Study of Heavy Drinking: Attitudes and Sociodemographic Factors in University Students. Journal of American College Health, 55, 83-89. doi:10.3200/JACH.55.2.83-90.

DeMartini, K. S., y Carey, K. B. (2012). Optimizing the Use of the AUDIT for Alcohol Screening in College Students. Psychological Assessment, 24, 954-963. doi:10.1037/ a0028519.

Encuesta sobre alcohol y drogas en población general en España (EDADES 2011) - 2012. (n.d.). http:/ /www.pnsd. msc.es/Categoria2/observa/pdf/EDADES2011.pdf.

Fleming, M. F., Barry, K. L., y MacDonald, R. (1991). The alcohol use disorders identification test (AUDIT) in a college sample. The International Journal of the Addictions, 26, 1173-1185. doi:10.3109/10826089109062153

Giménez, R., Badia, J., Baldomà, L., Vázquez, I., Serra, D., Cambras, T., y Rodamilans, M. (2013). Incorporación de un caso clínico transversal en el grado de Farmacia. Valoración de la experiencia docente en la asignatura de Bioquímica. FEM, 16, 1-68.

Hingson, R., Heeren, T., Winter, M., y Wechsler, H. (2005). Magnitude of alcohol-related mortality and morbidity among U.S. college students ages 18-24: changes from 1998 to 2001. Annual Review of Public Health, 26, 259-279. doi:10.1146/annurev.publhealth.26.021304.144652.

Karam, E., Kypri, K., y Salamoun, M. (2007). Alcohol use among college students: an international perspective. Current Opinion in Psychiatry, 20, 213-221. doi:10.1097/ YCO.0b013e3280fa836c.

Khadjesari, Z., Murray, E., Hewitt, C., Hartley, S. y Godfrey, C. (2011). Can stand-alone computer-based interventions reduce alcohol consumption? A systematic review. Addiction, 106, 267-282. doi:10.1111/j.1360-0443.2010.03214.x.

McCambridge, J., Bendtsen, M., Karlsson, N., White, I. R., Nilsen, P., y Bendtsen, P. (2013). Alcohol assessment and feedback by email for university students: main findings from a randomised controlled trial. The British Journal of Psychiatry, 203, 334-340. doi:10.1192/bjp.bp.113.128660. 
McCambridge, J., Bendtsen, P., Bendtsen, M., y Nilsen, P. (2012). Alcohol email assessment and feedback study dismantling effectiveness for university students (AMADEUS-1): study protocol for a randomized controlled trial. Trials, 13, 49.

Montaño, M., Morales, A., Gómez, M., Maldonado, A., y Gantiva, C. (2011). Consumo de alcohol en estudiantes universitarios. Psicogente, 14, 27-35.

Mota, N., Álvarez-Gil, R., Corral, M., Rodríguez-Holguín, S., Parada, M., Crego, A.,... Cadaveira, F. (2010). Risky alcohol use and heavy episodic drinking among Spanish University students: a two-year follow-up. Gaceta Sanitaria, 24, 372-377.

Observatorio Español de la droga y las toxicomanías. (2011). Informe. Recuperado de http://www.pnsd.msc.es/Categoria2/observa/pdf/oed2011.pdf.

Pengpid, S., Peltzer, K., van der Heever, H., y Skaal, L. (2013). Screening and brief interventions for hazardous and harmful alcohol use among university students in South Africa: results from a randomized controlled trial. International Journal of Environmental Research and Public Health, 10, 2043-2057. doi:10.3390/ijerph10052043.

Pérula de Torres, L. A., Fernández-García, J. A., Arias-Vega, R., Muriel-Palomino, M., Márquez-Rebollo, E., y Ruiz-Moral, R. (2005). Validación del cuestionario AUDIT para la identificación del consumo de riesgo y de los trastornos por el uso de alcohol en mujeres. Atención Primaria, 36, 499-506. doi:10.1016/S0212-6567(05)70552-7.

Reavley, N. J., Jorm, A. F., McCann, T. V, y Lubman, D. I. (2011). Alcohol consumption in tertiary education students. BMC Public Health, 11, 545. doi:10.1186/14712458-11-545.

Restrepo, L., Agudelo, A., Giraldo, M., y Sánchez, L. (2011). Factores de contexto asociados al consumo de alcohol en estudiantes universitarios. Revista CES Salud Pública, 2, 136-147.

Rodamilans, M., Gómez-Catalán, J., Piqué, E., Llobet, J., Gual, A., Giménez, R.,... Campanera, J. (2012). Desarrollo de casos clínicos transversales: experiencia dinamizadora de la Unidad Docente de Toxicología de la Universidad de Barcelona. Revista de Toxicolgía, 29, 141-146.

Rubio Valladolid, G., Bermejo Vicedo, J., Caballero Sánchez-Serrano, M. C., y Santo-Domingo Carrasco, J. (1998). Validación de la prueba para la identificación de trastornos por uso de alcohol (AUDIT) en atención primaria. Revista Clínica Española, 198, 11-14.

Sebena, R., Orosova, O., Mikolajczyk, R. T., y van Dijk, J. P. (2011). Selected sociodemographic factors and related differences in patterns of alcohol use among university students in Slovakia. BMC Public Health, 11, 849. doi:10.1186/1471-2458-11-849.

Seigers, D. K. L., y Carey, K. B. (2011). Screening and brief interventions for alcohol use in college health centers: a review. Journal of American College Health, 59, 151-158. doi :10.1080/07448481.2010.502199.

Slutske, W. S. (2005). Alcohol use disorders among US college students and their non-college-attending peers. Archives of General Psychiatry, 62, 321-327. doi:10.1016/ S0084-3717(08)70186-7.

Toumbourou, J., Stockwell, T., Neighbors, C., Marlatt, G., Sturge, J., y Rehm, J. (2007). Interventions to reduce harm associated with adolescent substance use. Lancet, 369,1391-1401. doi:10.1016/S0140-6736(07)60369-9.

Vinader-Caerols, C., Monleon, S. y Parra, A. (2014). Psycological and psycholicial effects of a high dose of alcohol in young men and women. Adicciones, 26, 238-246.

Wicki, M., Kuntsche, E., y Gmel, G. (2010). Drinking at European universities? A review of students' alcohol use. Addictive Behaviors, 35, 913-924. doi:10.1016/j.addbeh.2010.06.015.

Young, C. y de Klerk, V. (2008). Patterns of alcohol usage on a South African university campus: The findings of two annual drinking surveys. The African Journal of Drug and Alcohol Studies, 7, 101-112. 